\title{
Improving Revenue Management: A Real Option Approach
}

\author{
Wai-Ki Ching * $\quad$ Xun $\mathrm{Li}^{\dagger} \quad$ Tak-Kuen Siu ${ }^{\ddagger} \quad$ Zhen-Yu Wu ${ }^{\S}$
}

26 Jan. 2007

\begin{abstract}
In this article, we present a real option approach for improving revenue management regarding fluctuating commodity prices and time-varying strike prices in the field of operational research. We also take into account the cyclical nature of commodity prices, which is an important "stylised" fact in the empirical behavior of commodity prices. Two typical examples are provided to illustrate how real options can be used for enhancing profits and managing risk, which are important in revenue management. Valuation of these real options via a semi-analytical algorithm will be discussed.
\end{abstract}

Keywords: Air transport, energy, risk management, revenue management, real options, stochastic processes.

\section{Introduction}

Many firms from various industrial sectors face a common important problem of increasing or maximizing their revenues by selling a fixed stock of products or certain amount of services due to the limitation of the production or service capacity in a fixed time period. This is commonly known as the problem of revenue management. In many cases, those products or services will have a very low value or even zero value if they are not used by the end of the selling period. Typical examples of industries offering such products or services include airlines, hotels, rental cars and fashion goods. See the surveys by Belobaba (1987), Weatherford and Bodily (1992) and Geragthy and Johnson (1997) for detail. Some previous researches on revenue management mainly focus on the analysis of firms' pricing policy and selling strategy under the assumption that they are driving

\footnotetext{
*Advanced Modeling and Applied Computing Laboratory, Department of Mathematics, The University of Hong Kong, Pokfulam Road, Hong Kong. E-mail: wching@hkusua.hku.hk. Research supported in part by RGC Grants, HKU CRCG Grants, Hung Hing Ying Physical Sciences Research Fund and HKU Strategic Research Theme Fund on Computational Physics and Numerical Methods.

${ }^{\dagger}$ Department of Mathematics, National University of Singapore, Singapore 117543. E-mail: matlx@nus.edu.sg

${ }^{\ddagger}$ Department of Actuarial Mathematics and Statistics, School of Mathematical and Computer Sciences and the Maxwell Institute for Mathematical Sciences, Heriot-Watt University, Edinburgh EH14 4AS, UK.

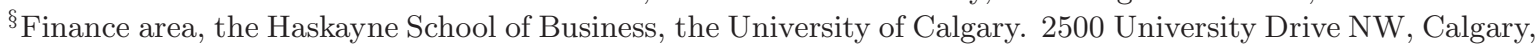
Alberta, Canada T2N 1N4. E-mail: zhenyu.wu@haskayne.ucalgary.
} 
force in price-setting actiing as monopolists in these industries (see, for example, Gallego and Van Ryzin (1994) and Feng and Gallengo (1995), and others). Under the assumption, a model with price certainty works well. However, in practice, the price of the underlying capacity or commodity is usually driven by market forces (See, for example, Deng, Feng and $\mathrm{Li}$ (2006) for discussions). This makes the model with price certainty not satisfactory. When the price of the underlying capacity or commodity is fluctuating due to changes in economic factors, revenue management not only plays an important role for enhancing or maximizing profit, but also plays a significant role for risk management. This extends the domain of revenue management from enhancing or maximizing profit to both managing risk and enhancing or maximizing profit. Traditionally, the main focus of the literature on revenue management concerns profit enhancement or profit maximization for industries, such as the airline industry and the car rental industry, with fluctuating commodity prices (see, for example, Anderson, et al., 2004). There is a limited amount of literature concerning the issue of risk management in revenue management when the price of the underlying commodity or capacity fluctuates randomly over time.

In this paper, we shall address the two important aspects, namely, profit enhancement and risk management, in revenue management. In particular, we shall apply a special type of options written on the underlying commodities to achieve the goals of profit enhancement and risk managment in revenue management. We shall consider two typical industrial examples in revenue management to illustrate how the two goals can be achieved through the use of the options. First, an example from the airline industry is provided for studying profit enhancement. The price of the airticket fluctuates randomly over time due to market forces, economic conditions and travel seasons. In reality, many travellers buy airline ticket from travel agents, which play an important role as an intermediation between airline companies and travellers. Here, we introduce a callable ticket or a discount ticket sold by a travel agent to a traveller, which gives the travel agent the right to buy back or call back the ticket at the last minute at which the agent can still sell the ticket. Since the travel agent has the right to buy back the ticket, the travel agent have to sell the callable ticket at a lower price compared with the original ticket. Travellers who do not have definite or exact travel plans may consider buying callable tickets. We shall consider the valuation of these discounted tickets. Besides determining the price of a discount ticket, it is an important practical problem about the determination of the optimal time at which the agents should buy back the ticket in order to maximize their payoffs or profits. We shall determine the optimal time by solving an optimal stopping problem. The second example based on Strategic Petroleum Reserve (SPR) in the energy industry is to examine the risk management issue in revenue management. Since the SPR producers intend to hedge risk borne by them due to the special nature of energy commodities, they can also use long call options while selling the energy commodities to the SPR builders. To provide more flexibility, we consider both the European-style and American-style energy options. Throughout this paper, we consider a special type of options with time-varying strike 
prices to enhance the profits or achieve better risk management results. We incorporate this special feature to our real option-based revenue management models and price the options using a semi-analytical algorithm, which provides practitioners with a much more convenient way to value the real options compared with some numerical methods, such as the finite-difference method. To illustrate the estimation accuracy of our approach, we also provide numerical examples and compare the approximated results with those obtained from the finite-difference method. Results show that our semi-analytical approach does not deteriorate the accuracy of option pricing.

The rest of this paper is structured in the sequel. Section 2 provides a review on some relevant literature. A general problem setting for the real option with a time-varying strike price is briefly presented in Section 3. An example from the airline industry for profit enhancement is presented in Section 4, followed by Section 5 which provides an example of SPR from the energy industry for examining the risk-management issues. Concluding remarks and further implications are included in Section 6.

\section{Literature Review}

Recently, techniques for real option valuation have been applied to investigate the decision making in this field in the presence of uncertainty (Anderson et al., 2004). In the traditional financial economics literature, effects of random fluctuation in spot prices and limited capacity are often ignored in studies on options contracts and real options (Allaz and Vila, 1993) for analytical simplicity. In particular, revenue management on callable products has received considerable interest, and some previous studies (e.g., Gallego, Kou and Phillips, 2004; Feng and Gallego, 2005) have made significant contributions to this literature.

As one of the pioneer studies in this literature, Wu and Kleindorfer (2005) incorporate option-based contracts in supply management into a game-theoretical model and focus on electricity markets. As discussed by Gallego, et al. (2004), in addition, the nature of callable products provides us with a different angle to examine revenue management. However, the issues of using callable products to enhance profit profiles and to improve the practice of risk management have not been extensively explored yet.

More interestingly, one realizes that one of the most important features of the underlying commodities in revenue management is their price fluctuation over time (Anderson, et al., 2004; Gallego, et al., 2004; Feng and Gallego, 2005). Thus, real options need to have time-varying strike prices so as to enhance profits and/or to hedge risk.

Multiple contributions are made to the literature by this paper. Firstly, we provide a new real option approach to deal with both of the issues of profit enhancement and risk management, which are important in the revenue management. Secondly, we highlight the special feature of fluctuating commodity prices in revenue management and adopt an effective semi-analytical algorithm for pricing real options with time-varying strike 
prices. Thirdly, it sheds lights on the risk management issues in energy financing and risk hedging using real options. Finally, it provides testable implications for the airline industry and for energy finance and economics regarding the demand-supply equilibrium in building strategic petroleum reserve. This is mainly because changes in demand and/or supply influenced by many factors in the economy may affect the profit-enhancement and risk-management strategies significantly.

\section{A General Problem Setting for Real Options with Time-Varying Strike Prices}

To construct a real option with a time-varying strike price, we denote $K_{t}$ as the strike price depending on time $t$. For analytical simplicity, we define $K_{t}=K e^{r t}$, where $K$ is pre-determined in the options contract and $r$ is a constant risk-free rate.

We suppose there is an underlying commodity $S$ with spot price $S_{t}$ at time $t$. The spot price of $S$ fluctuates randomly over time according to certain stochastic process which will be specified in the next two sections for different commodities. A call option written on $S$, with time-varying strike $K_{t}$ and maturity $T$ has a discounted payoff $e^{-r T}\left(S_{T}-K_{T}\right)^{+}$. Note that the option is a European option if it is not allowed to be exercised before the maturity $T$; otherwise, it is an American option, and there could be an optimal early exercise time $\tau^{*} \in(0, T]$. The early exercise feature of the American option with time-varying strike poses additional difficulty in its pricing issue.

Wu, Kleindorfer, Sun and Zhang (2002) present a model for pricing capacity options on non-storeable goods, and Xia and Zhou (2005), a pioneer study in option valuation, provide a closed-form formula for pricing perpetual American options with time-varying strike prices. Whereas Xia and Zhou (2005) pave the road for further research on this topic, exploring the use of options with both time-varying strike prices and finite maturities in revenue management and valuing these options have not been explored in the literature. It is of practical relevance to explore the use of these options to improve revenue management when the underlying commodity prices are fluctuating and/or capacity is limited.

In the next two sections, we explore the use of these options in the airline industry and the energy sector and consider the valuation of these options. We shall adopt a semianalytical algorithm for pricing these options on commodities with fluctuating prices in the airline industry and in the energy industry, respectively.

\section{Profit Enhancement: An Example from the Airline Industry}

In the airline industry, capacity is limited and therefore revenue management, including both the determination of prices over time and booking process, plays a key role in airline companies' profits. Previous studies (e.g., Gallego, et al., 2004) treat airline tickets as 
callable products for revenue management and discuss some alternatives such as bumping, flexible products and last-minute discounts.

In this section, we present a real option-based revenue management model with a call option purchased by travel agents, and the main objective is to enhance the agents' profits by purchasing call options from their customers while selling them air tickets. The call option premium appears in the form of a discount off the regular airfare. Customers who are flexible regarding all aspects of their trips and have the possibility of changing their travel plans are willing to accept the deal. In this case, travel agents can call back these tickets when some other customers need to travel with necessity and/or purchase air tickets shortly before departure at much higher prices. This can enhance the profits of the agents.

To illustrate the real option approach in this model, we assume that a customer purchases an air ticket at a discounted price $c S_{0}$ at time $t_{0}$, where $0<c<1$. $S_{0}$ is the regular airfare. At the same time, the deal is that with the call option, the travel agent can buy back the ticket at the price $K_{t}$ at any time $t \in(0, T]$, where $T$ is the last minute at which the agent can sell the ticket and $T$ represents the maturity of the embedded call option.

As defined in Section 2, the time-varying strike price $K_{t}=S_{0} e^{r t}$ and $S_{t}$ is the spot price of the air ticket at time $t$. Suppose $C_{0}$ is the price of a call option written on the ticket $S$, with strike $K_{t}$ and maturity at time $T$. The discount coefficient $c$ can be determined by:

$$
(1-c) S_{0}=C_{0}
$$

Furthermore, since airfare has the property of seasonality, we introduce an additional factor to the model to describe it following Elliott, Sick and Stein (2003). Thus, the spot price of the air ticket under a real-world probability $\mathcal{P}$ follows:

$$
S_{t}=S_{0} g_{t} e^{X_{t}}
$$

where $g_{t}$ is the seasonality factor defined as

$$
g_{t}=e^{\delta \int_{0}^{t} \sin (2 \pi s+\xi) d s}
$$

and

$$
d X_{t}=(\mu-q) d t+\sigma d W_{t}
$$

Here $\mu$ is the long-run mean return, $q$ is a dividend-like term, $\sigma$ is volatility, and $W_{t}$ is a standard Brownian motion. In general, one can consider that parameters $r, \mu, q$, $\sigma$, and $S_{t}$ are deterministic functions of time $t$ following prior research (e.g., Duffie and Richardson, 1991), and therefore, for analytical simplicity, we assume they are constant. A more general case can be extended easily by following the same method presented in the following. 
By Itô's lemma, we have

$$
\begin{aligned}
d S_{t} & =\left(\delta \sin (2 \pi t+\xi)+\mu-q+\frac{1}{2} \sigma^{2}\right) S_{t} d t+\sigma S_{t} d W_{t} \\
& =\left(b_{t}-q\right) S_{t} d t+\sigma S_{t} d W_{t}
\end{aligned}
$$

where $b_{t}=\delta \sin (2 \pi t+\xi)+\mu+\frac{1}{2} \sigma^{2}$.

For pricing options, we need to work in a risk-neutral world. First, we suppose that there is a constant market price of risk $\lambda$. Let $\mathcal{F}_{t}$ denote the information set generated by the values of the Brownian motion $W$ up to and including time $t$. Define a risk-neutral measure $\mathcal{Q}$ equivalent to $\mathcal{P}$ on $\mathcal{F}_{T}$ by the following Radon-Nikodym derivative:

$$
\left.\frac{d \mathcal{Q}}{d \mathcal{P}}\right|_{\mathcal{F}_{T}}:=\exp \left(-\lambda W_{T}-\frac{1}{2} \lambda^{2} T\right) .
$$

From Girsanov's theorem,

$$
W_{t}^{\dagger}:=\lambda t+W_{t}
$$

is a standard Brownian motion with respect to $\left\{\mathcal{F}_{t}\right\}$ under $\mathcal{Q}$.

Let $\mu^{\dagger}:=\mu-\lambda \sigma$. Write $b_{t}^{\dagger}:=\delta \sin (2 \pi t+\xi)+\mu^{\dagger}+\frac{1}{2} \sigma^{2}$. Then the risk-neutral dynamic of $S$ under $\mathcal{Q}$ is:

$$
d S_{t}=\left(b_{t}^{\dagger}-q\right) S_{t} d t+\sigma S_{t} d W_{t}^{\dagger}
$$

\subsection{Discounted Airfare with A European Option}

We first present the fair value of the discounted airfare when a European call option is purchased by a travel agent. The European call option gives the agent the right, but not the obligation, to buy or call back the ticket. However, the agent cannot exercise the option before its maturity $T$. We can determine the value of the European option, given that

$$
(1-c) S_{0}=e^{-r T} E\left[\left(S_{T}-K_{T}\right)^{+} \mid S_{0}\right],
$$

where $E$ is the expectation with respect to the risk-neutral probability $\mathcal{Q}$ defined above.

Note that the discount $c$ offers to customers by the agent can be determined completely once we have determined the premium of the European option with payoff $\left(S_{T}-K_{T}\right)^{+}$at the maturity $T$. Hence, we first consider the following problem according to risk-neutral spot price (4.4):

$$
C(t, S)=e^{-r(T-t)} E\left[\left(S_{T}-K_{T}\right)^{+} \mid S_{t}=S\right]
$$

In the above European option, $K_{T}$ is used only at the maturity and regarded as fixed. 
Then the above (4.5) satisfies the partial differential equation (PDE):

$$
\left\{\begin{array}{l}
\frac{\partial C}{\partial t}+\frac{1}{2} \sigma^{2} S^{2} \frac{\partial^{2} C}{\partial S^{2}}+\left(b_{t}^{\dagger}-q\right) S \frac{\partial C}{\partial S}-r C=0 \\
C(T, S)=\left(S-K_{T}\right)^{+}
\end{array}\right.
$$

As shown in Wilmott, Howison and Dewynne (1996), solving equation (4.6) yields

$$
\left\{\begin{array}{l}
C(t, S)=S_{0} e^{\int_{t}^{T}\left(b_{s}^{\dagger}-q-r\right) d s} N\left(d_{1}(t, S)\right)-K e^{r t} N\left(d_{2}(t, S)\right) \\
d_{1}(t, S)=\frac{\ln (S)-\ln \left(K e^{r T}\right)+\int_{t}^{T}\left(b_{s}^{\dagger}-q+\frac{1}{2} \sigma^{2}\right) d s}{\sigma \sqrt{T-t}} \\
d_{2}(t, S)=d_{1}(t, S)-\sigma \sqrt{T-t}
\end{array}\right.
$$

Hence, the discount factor $c$ is expressed by

$$
c=1-e^{\int_{0}^{T}\left(b_{s}^{\dagger}-q-r\right) d s} N\left(d_{10}\right)-K N\left(d_{20}\right) / S_{0},
$$

where

$$
\left\{\begin{array}{l}
d_{10}=\frac{\ln \left(S_{0}\right)-\ln \left(K e^{r T}\right)+\int_{0}^{T}\left(b_{s}^{\dagger}-q+\frac{1}{2} \sigma^{2}\right) d s}{\sigma \sqrt{T}}, \\
d_{20}=d_{10}-\sigma \sqrt{T}
\end{array}\right.
$$

\subsection{Discounted Airfare with An American Option}

However, the travel agent can have more flexibility if he relaxes the restrictions in the above European option by longing an American option instead. In this case, both the optimal exercise time $\tau^{*}$ and the value of $c$, where

$$
(1-c) S_{0}=E\left[e^{-r \tau^{*}}\left(S_{\tau^{*}}-K_{\tau^{*}}\right)^{+}\right]
$$

need to be determined.

We now consider the following optimal stopping problem, which is different from pricing a standard American call option since the strike price is time-varying. At time $t$, the American call option price is

$$
C^{A}(t, S)=\sup _{\tau \in \mathcal{T}_{t, T}} E\left[e^{-r(\tau-t)}\left(S_{\tau}-K_{\tau}\right)^{+} \mid S_{t}=S\right]
$$

Once $C^{A}(t, S)$ is determined, we can determine the value of the discount factor $c$. Note that the optimal exercise time $\tau^{*}$ is the same for $C^{A}(t, S)$ and $c$. 
The above (4.9) satisfies the following free-boundary PDE problem:

$$
\left\{\begin{array}{l}
\frac{\partial C}{\partial t}+\frac{1}{2} \sigma^{2} S^{2} \frac{\partial^{2} C}{\partial S^{2}}+\left(b_{t}^{\dagger}-q\right) S \frac{\partial C}{\partial S}-r C \leq 0 \\
C(t, S)-\left(S_{t}-K_{t}\right)^{+} \geq 0 \\
\left(\frac{\partial C}{\partial t}+\frac{1}{2} \sigma^{2} S^{2} \frac{\partial^{2} C}{\partial S^{2}}+\left(b_{t}^{\dagger}-q\right) S \frac{\partial C}{\partial S}-r C\right)\left(C(t, S)-\left(S_{t}-K_{t}\right)^{+}\right)=0 \\
C(T, S)=\left(S-K_{T}\right)^{+}
\end{array}\right.
$$

or

$$
\left\{\begin{array}{l}
\min \left\{-\frac{\partial C}{\partial t}+\frac{1}{2} \sigma^{2} S^{2} \frac{\partial^{2} C}{\partial S^{2}}-\left(b_{t}^{\dagger}-q\right) S \frac{\partial C}{\partial S}+r C, C(t, S)-\left(S_{t}-K_{t}\right)^{+}\right\}=0 \\
C(T, S)=\left(S-K_{T}\right)^{+}
\end{array}\right.
$$

Since both American and European options satisfy the same PDE, so does the early exercise premium

$$
\epsilon(t, S):=C^{A}(t, S)-C(t, S)
$$

Thus, $\epsilon(t, S)$ satisfies

$$
\frac{\partial \epsilon}{\partial t}+\frac{1}{2} \sigma^{2} S^{2} \frac{\partial^{2} \epsilon}{\partial S^{2}}+\left(b_{t}^{\dagger}-q\right) S \frac{\partial \epsilon}{\partial S}-r \epsilon=0 .
$$

We approximate the solution for the above PDE by assuming that $\epsilon(t, S)$ is approximately given by the following separated form:

$$
\epsilon(t, S) \approx H(S, h) h(t)
$$

Then

$$
h \frac{\partial H}{\partial h} \frac{\partial h}{\partial t}+H \frac{\partial h}{\partial t}+\left(b_{t}^{\dagger}-q\right) S h \frac{\partial H}{\partial S}+\frac{1}{2} \sigma^{2} S^{2} h \frac{\partial^{2} H}{\partial S^{2}}-r H h=0 .
$$

We further assume that

$$
h(t)=1-e^{-r(T-t)},
$$

and therefore,

$$
\frac{\partial h}{\partial t}=r(h-1)
$$

With appropriate substitutions and variable changes, this gives

$$
S^{2} \frac{\partial^{2} H}{\partial S^{2}}+\beta_{t} S \frac{\partial H}{\partial S}-\frac{\alpha}{h} H=(1-h) \alpha \frac{\partial H}{\partial h},
$$


where

$$
\alpha=\frac{2 r}{\sigma^{2}} \quad \text { and } \quad \beta_{t}=\frac{2\left(b_{t}^{\dagger}-q\right)}{\sigma^{2}} .
$$

Equation (4.15) can be approximated by

$$
S^{2} \frac{\partial^{2} H}{\partial S^{2}}+\beta_{t} S \frac{\partial H}{\partial S}-\frac{\alpha}{h} H=0
$$

since the right-hand side of Equation (4.15) is generally fairly small and can be ignored. When $T-t$ is large, $1-h$ is close to zero; when $T-t$ is small, on the other hand, $\frac{\partial H}{\partial h}$ is close to zero. Solving equation (4.16) and applying boundary conditions lead to

$$
C^{A}(t, S)= \begin{cases}C(t, S)+A_{t}\left(\frac{S}{S^{*}}\right)^{\gamma_{t}}, & \text { if } S<S^{*}, \\ S-K e^{r t}, & \text { if } S \geq S^{*},\end{cases}
$$

where

$$
A_{t}=\frac{S^{*}}{\gamma_{t}}\left[1-e^{b_{t}^{\dagger}-q-r} N\left(d_{1}\left(t, S^{*}\right)\right)\right] \quad \text { and } \quad \gamma_{t}=\frac{1}{2}\left(-\left(\beta_{t}-1\right)+\sqrt{\left(\beta_{t}-1\right)^{2}+\frac{4 \alpha}{h}}\right) .
$$

The variable $S^{*}$ is the critical stock price above which the option should be exercised. It can be estimated by solving the equation

$$
S^{*}-K e^{r t}=C\left(t, S^{*}\right)+A_{t}
$$

iteratively.

In short, travel agents can be better off if they long options while selling air tickets. The prices of the call options appear in the form of discounts off the regular airfare. However, it is worth noting that this example is not exactly the same as that in the revenue management in prior research, since we focus on an intermediate party in the airline industry.

\subsection{Numerical Examples}

In this subsection, we use numerical examples for the European and American call options embedded in the discounted airfare to further illustrate profit maximization in revenue management of the airline industry described in Sections 4.1 and 4.2. Assuming that

$$
r=0.05, q=0.08, \mu^{\dagger}=0.065, \xi=1, \delta=0.01, \sigma=0.3
$$

we price the embedded options and the discounted airfare for various values of initial spot price $S_{0}$ and maturity $T$. We suppose that $T$ takes values 0.25 year, 0.5 year, 0.75 year and 1 year, respectively, and $S_{0}$ ranges from 100 to 140 with an increment of 10 . The figures we have assumed are consistent with the corresponding figures in the industries. For instance, the spot price of the ticket can be from $£ 100$ to $£ 140$, which are compatiable 
with the prices of tickets for UK internal flights or tickets from UK to other EU regions. The maturity $T$ from 0.25 year and 1 year is also compatible with the real time duration for a ticket, which is available for sale.

We shall compare the option prices obtained from the semi-analytical algorithm with those from the finite difference scheme. Since there is no closed-form solution to the pricing problem we considered above, one might use some numerical method, such as the finite-difference scheme to solve the pricing P.D.E.s. The finite-difference scheme has the advantage of giving a good approximate pricing result when an appropriate or a fine grid for discretizing the space and the time domains of the pricing P.D.E.s. is used. However, the computational time and effort required to implement the finite-difference scheme can be substantial. The semi-analytical algorithm we proposed can provide a convenient way to determine the option prices. We shall demostrate the accuracy of the semi-analytical algorithm by comparing it with a finite-difference scheme with a fine grid, which can deliver accurate approximation results.

Tables 1 presents the European and the American call option prices obtained from the BAW quadratic approximation [3] when the initial price of underlying commodity and the maturity take different values, respectively. Comparing the option prices obtained from the above semi-analytical algorithm with those from the finite difference scheme, we find that the relative errors are negligible. Corresponding values of the discounted airfares are also presented in Table 1, and we find that the approximation algorithm provides accurate estimation and is easy to implement.

\section{Risk Management: An Example from the Energy Industry}

In the energy industry, revenue management is also one of the critical issues. Strategic petroleum reserve (SPR) has been a hot issue for three decades, and it becomes one of the crucial factors boosting the prices of natural resources recently. In this section, we use SPR to illustrate the risk-management issues, in particular the risk-hedging issues, in real option-based revenue management, and investigate these issues from the producer side. Considering the special features of energy commodities, such as seasonality and meanreverting prices, we again price both European and American options with time-varying strike prices.

\subsection{European and American Options on Energy Commodities}

To hedge risk, producers of energy products can long call options while selling underlying commodities to the SPR builders. Thus, we shall focus on European and American call options in this section. Some special natures of the energy commodities significantly affect the valuations of options on them, and therefore need to be pointed out here.

First, due to the fluctuation of energy commodity prices and the existence of time value of money as shown in the traditional finance literature, the strike price of the relevant 
options should be time-varying to better hedge the risk, and can again be defined as $K_{t}=K e^{r t}, t \in(0, T]$ as before, where $K$ is usually taken to be $S_{0}$. If they are European options, $K_{T}=K e^{r T}$, and they are equivalent to regular European options when the risk-free rate $r$ is constant. If they are American options, however, the factor $t$ plays an important role in determining the option prices. Given that the spot price of an underlying energy commodity is $S_{0}$ at time $t_{0}$, for analytical simplicity, we define the energy commodity price producers sell at is $S_{0}-C_{0}$ at time $t_{0}$, where $C_{0}$ is the call option premium.

Second, due to the features of seasonality and the mean-reverting property of energy commodity prices, we assume that under a real-world probability $\mathcal{P}$, the spot price of the underlying energy commodity follows $S_{t}=S_{0} g_{t} e^{X_{t}}$, where $g_{t}$ is again the seasonality factor defined as $g_{t}=e^{\delta \int_{0}^{t} \sin (2 \pi s+\xi) d s}$ and

$$
d X_{t}=\kappa\left(\gamma-X_{t}\right) d t+\sigma d W_{t}
$$

$\kappa$ is the strength of mean-reversion, which determines the speed of adjustment pulling the randomly moving value of the mean-reverting asset toward a central location $\gamma$, and $\gamma$ is also referred to the long-run mean return.

By Itô's lemma, we get

$$
\begin{aligned}
d S_{t} & =\left(\delta \sin (2 \pi t+\xi)+\kappa \gamma+\frac{1}{2} \sigma^{2}-\kappa \ln S_{t}\right) S_{t} d t+\sigma S_{t} d W_{t} \\
& =\left(\bar{b}_{t}-\kappa \ln S_{t}\right) S_{t} d t+\sigma S_{t} d W_{t}
\end{aligned}
$$

where $\bar{b}_{t}=\delta \sin (2 \pi t+\xi)+\kappa \gamma+\frac{1}{2} \sigma^{2}$.

For pricing options on the energy commodity, we also need to consider a risk-neutral world. We suppose that there is a constant market price of risk $\bar{\lambda}$. Define a risk-neutral measure $\overline{\mathcal{Q}}$ equivalent to $\mathcal{P}$ on $\mathcal{F}_{T}$ by the following Radon-Nikodym derivative:

$$
\left.\frac{d \overline{\mathcal{Q}}}{d \mathcal{P}}\right|_{\mathcal{F}_{T}}:=\exp \left(-\int_{0}^{T} \bar{\lambda} \ln S_{t} d W_{t}-\frac{1}{2} \int_{0}^{T} \bar{\lambda}^{2}\left(\ln S_{t}\right)^{2} d t\right) .
$$

¿From Girsanov's theorem,

$$
\bar{W}_{t}^{\dagger}:=W_{t}+\int_{0}^{t} \bar{\lambda} \ln S_{u} d u
$$

is a standard Brownian motion with respect to $\left\{\mathcal{F}_{t}\right\}$ under $\mathcal{Q}$.

Let $\kappa^{\dagger}:=\kappa+\bar{\lambda} \sigma$. Then, under $\mathcal{Q}$, the risk-neutral dynamic of $S$ is:

$$
d S_{t}=\left(\bar{b}_{t}-\kappa^{\dagger} \ln S_{t}\right) S_{t} d t+\sigma S_{t} d \bar{W}_{t}^{\dagger}
$$


At time $t$, thus, the American call option price is

$$
C^{A}(t, S)=\operatorname{esssup}_{\tau \in \mathcal{T}_{t, T}} E\left[e^{-r(\tau-t)}\left(S_{\tau}-K_{\tau}\right)^{+} \mid S_{t}=S\right]
$$

which satisfies the following free-boundary PDE problems:

$$
\left\{\begin{array}{l}
\frac{\partial C}{\partial t}+\frac{1}{2} \sigma^{2} S^{2} \frac{\partial^{2} C}{\partial S^{2}}+\left(\bar{b}_{t}-\kappa^{\dagger} \ln S\right) S \frac{\partial C}{\partial S}-r C \leq 0 \\
C(t, S)-\left(S_{t}-K_{t}\right)^{+} \geq 0 \\
\left(\frac{\partial C}{\partial t}+\frac{1}{2} \sigma^{2} S^{2} \frac{\partial^{2} C}{\partial S^{2}}+\left(\bar{b}_{t}-\kappa^{\dagger} \ln S\right) S \frac{\partial C}{\partial S}-r C\right)\left(C(t, S)-\left(S_{t}-K_{t}\right)^{+}\right)=0 \\
C(T, S)=\left(S-K_{T}\right)^{+}
\end{array}\right.
$$

\subsection{Numerical Examples}

To further illustrate the implementation of the algorithm introduced in Section 4.1, we consider the following numerical experiments using some specimen values for the model parameters such as $r=5 \% ; \kappa=0.15, \gamma=0.5, \xi=1, \delta=0.01, \bar{\lambda}=0.06$ and $\sigma=0.3$. For different values of the initial price of the energy commodity $S_{0}$ and the time to maturity $T$, we price European and American call options purchased by energy commodity producers, and calculate their corresponding real energy commodity prices defined by $S_{0}-C_{0}$. We suppose that $S_{0}$ ranges from 30 to 70 with an increment of 10 , while $T$ can be $0.25,0.5$, 0.75 and 1 years. Table 2 presents the numerical results for the European and American call option prices approximated by the finite-difference scheme, and their corresponding real commodity prices.

Note that we have assumed zero dividend payment in the numerical examples, and therefore, according to the standard finance theory, the American call option prices should have been the same as the corresponding European counterparts due to the absence of dividend payment. However, this is not the truth here since the strike prices of the American call options are time-varying. This makes their behaviors totally different from those predicted from the standard option pricing theory. We can see that the gaps between the American call option prices and the European ones are quite substantial, and they increase in the length of maturity. In other words, an early exercise premium for the American call option is present due to the presence of the time-varying-strike-price (TVSP) effect, and this illustrates that the TVSP effect on option premium is significant.

One noticeable feature of the embedded European and American call options is that the strike price depends on both the initial price of the energy commodity $S_{0}$ and the time to maturity $T$. This makes the behaviors of call option prices as functions of the initial underlying price $S_{0}$ different from those of the standard call option prices with constant strike prices. This difference becomes more pronounced when we consider a European call option with longer time to maturity, and it is mainly due to the feature of seasonality.

As shown in Table 2, the European call option prices decrease in the initial price of 
the underlying energy commodity when $T=0.75$ year and when the maturity is one year. Please note that this does not violate the features of European call option prices defined in the Black-Scholes formula since the underlying security in the Black-Scholes model follows a log-normal process. The phenomena shown in our numerical experiments reflect the special feature, seasonality, of energy commodities. We also find that the influence of seasonality does not appear when call options are of the American style, and this is mainly because of the self-adjustment made by the option holders when they can choose an optimal early exercise time.

In short, we find that the seasonality have significant effects on European call option prices, but insignificant effects on the American ones. On the contrary, the TVSP effect is significant when call options are of the American style, but insignificant when they are of the European style.

\section{Concluding Remarks and Further Implications}

While revenue management has attracted serious attention of academic researchers in various disciplines and practitioners in different sectors, profit maximization has been the major focus of previous studies. Although more and more people have realized the importance of the risk-management issues, in particular the risk-hedging issues, in this field, they have not been extensively studied in the literature. This study introduces a real-option approach to deal with both profit enhancement and risk hedging issues in revenue management.

On the basis of studies on callable products in revenue management (e.g., Gallego, et al., 2004; Feng and Gallego, 2005), we used two typical examples in two industries, the airline industry and the energy industry, to illustrate the proposed approach. Due to the special features of airline tickets and energy commodities, in addition, we incorporate the seasonality and TVSP effects into the models, and propose a semi-analytical algorithm for pricing the real options. It develops the approach presented in Xia and Zhou (2005) which gives a closed-form expression for pricing perpetual American options with time-varying strike prices. Numerical examples are also presented to illustrate the estimation accuracy of the proposed approach by comparing with results obtained from the finite difference scheme.

In the airline industry, travel agents can buy call options from their customers while selling air tickets so as to be able to call back these tickets if they can sell them at much higher prices. To enhance their own profits, in other words, travel agents long call options in order to purchase the tickets back from their customers if some other customers travel with necessity and would like to pay higher prices. Customers who are flexible regarding their trips and those who can change their travel plans are willing to accept the deal. The option premium appears in the form of a discount offered to the customers when they purchase air tickets. In the energy industry, strategy petroleum reserve (SPR) has been 
a hot topic for at least 30 years, and it is one of the most significant factors boosting the prices of energy commodities. To hedge risk, producers of energy commodities can long call options from the strategic reserve builders while selling them underlying products. While constructing the models for these two industries, we find that the seasonality effect is significant in both of them while the mean-reversion effect is significant in the energy industry as documented in the literature.

Using the models constructed on the basis of real options and incorporating seasonality, mean-reversion and time-varying strike prices into the models, we examine both aspects of revenue management, profit enhancement and risk management. Besides that we show the effectiveness and the estimation accuracy of the proposed approach, we also find that seasonality has significant effects only on European call prices, but the time-varyingstrike-price effects are significant only on the American ones, in the energy industry.

\section{References}

[1] Anderson C K, Davison M and Rasmussen H (2004). Revenue management: a real options approach. Naval Research Logistics. 51: 686-703.

[2] Allaz B and Vila J L (1993). Cournot competition, forward markets and efficiency. Journal of Economic Theory. 59: 1-16.

[3] Barone-Adesi G. and Whaley R (1987). Efficient analytic approximation of American option values. Journal of Finance, 42 1-20.

[4] Duffie D and Richardson H R (1991). Mean-variance hedging in continuous time. The Annals of Applied Probability. 1: 1-15.

[5] Elliott R J, Sick G A and Stein M (2003). Modeling electricity price risk. Working paper, University of Calgary and University of Oregon.

[6] Feng Y and Gallego G (2005). Optimal booking control with a callable booking class. Working paper, Chinese University of Hong Kong and Columbia University in the City of New York.

[7] Gallego G, Kou S G and Phillips R (2004). Revenue management of callable products. Working paper, Columbia University.

[8] Wilmott P, Howison S and Dewynne J (1996). The Mathematics of Financial Derivatives. Cambridge University Press, USA.

[9] Wu D J and Kleindorfer P R (2005). Competitive options, supply contracting, and electronic markets. Management Science. 51: 452-466.

[10] Wu D J, Kleindorfer P R, Sun Y and Zhang J E (2002). The price of a real option on capacity. Working paper, Drexel University, University of Pennsylvania, and Hong Kong University of Science and Technology. 
[11] Xia J and Zhou X (2005). Stock loans. To appear in Mathematical Finance.

\begin{tabular}{|c|c|c|c|c|c|c|}
\hline$S_{0}$ & $\begin{array}{c}\text { European } \\
\text { (Fin. Diff.) }\end{array}$ & $\begin{array}{c}\text { European } \\
\mathrm{Eq}(4.7) \\
\end{array}$ & $\begin{array}{c}\text { Discounted } \\
\text { Airfare }\end{array}$ & $\begin{array}{c}\text { American } \\
\text { (Fin. Diff.) }\end{array}$ & $\begin{array}{l}\text { American } \\
\mathrm{Eq}(4.17)\end{array}$ & $\begin{array}{c}\text { Discounted } \\
\text { Airfare } \\
\end{array}$ \\
\hline \multicolumn{7}{|c|}{$T=0.25$ year } \\
\hline 100 & 3.5359 & 3.5357 & 96.4643 & 3.6015 & 3.6125 & 96.3875 \\
\hline 110 & 3.8892 & 3.8892 & 106.1108 & 3.9614 & 3.9738 & 106.0262 \\
\hline 120 & 4.2422 & 4.2428 & 115.7572 & 4.3211 & 4.3350 & 115.6650 \\
\hline 130 & 4.5965 & 4.5963 & 125.4037 & 4.6819 & 4.6963 & 125.3037 \\
\hline 140 & 4.9503 & 4.9499 & 135.0501 & 5.0422 & 5.0576 & 134.9424 \\
\hline \multicolumn{7}{|c|}{$T=0.5$ year } \\
\hline 100 & 4.6123 & 4.6113 & 95.3887 & 4.8304 & 4.8441 & 95.1559 \\
\hline 110 & 5.0742 & 5.0724 & 104.9276 & 5.3140 & 5.3285 & 104.6715 \\
\hline 120 & 5.5350 & 5.5336 & 114.4664 & 5.7968 & 5.8129 & 114.1871 \\
\hline 130 & 5.9968 & 5.9947 & 124.0053 & 6.2802 & 6.2973 & 123.7027 \\
\hline 140 & 6.4577 & 6.4558 & 133.5442 & 6.7631 & 6.7817 & 133.2183 \\
\hline \multicolumn{7}{|c|}{$T=0.75$ year } \\
\hline 100 & 5.2358 & 5.2351 & 94.7649 & 5.6326 & 5.6848 & 94.3152 \\
\hline 110 & 5.7594 & 5.7586 & 104.2414 & 6.1959 & 6.2532 & 103.7468 \\
\hline 120 & 6.2828 & 6.2821 & 113.7179 & 6.7591 & 6.8217 & 113.1783 \\
\hline 130 & 6.8063 & 6.8056 & 123.1944 & 7.3223 & 7.3902 & 122.6098 \\
\hline 140 & 7.3302 & 7.3291 & 132.6709 & 7.8858 & 7.9587 & 132.0413 \\
\hline \multicolumn{7}{|c|}{$T=1$ year } \\
\hline 100 & 5.7857 & 5.7870 & 94.2130 & 6.2690 & 6.4255 & 93.5745 \\
\hline 110 & 6.3643 & 6.3656 & 103.6344 & 6.8960 & 7.0680 & 102.9320 \\
\hline 120 & 6.9430 & 6.9443 & 113.0557 & 7.5229 & 7.7106 & 112.2894 \\
\hline 130 & 7.5216 & 7.5230 & 122.4770 & 8.1499 & 8.3531 & 121.6469 \\
\hline 140 & 8.1002 & 8.1017 & 131.8983 & 8.7768 & 8.9957 & 131.0043 \\
\hline
\end{tabular}




\begin{tabular}{|c|c|c|c|c|}
\hline \multicolumn{5}{|c|}{ Table 2 European and American Option Prices in the Energy Industry } \\
\hline$S_{0}$ & $\begin{array}{c}\text { European }\left(C_{0}\right) \\
\text { (Finite Difference) }\end{array}$ & $\begin{array}{c}\text { Real Commodity } \\
\text { Price }\left(S_{0}-C_{0}\right)\end{array}$ & $\begin{array}{c}\text { American }\left(C_{0}\right) \\
\text { (Finite Difference) }\end{array}$ & $\begin{array}{c}\text { Real Commodity } \\
\text { Price }\left(S_{0}-C_{0}\right)\end{array}$ \\
\hline \multicolumn{5}{|c|}{$T=0.25$ year } \\
\hline 30 & 0.4903 & 29.5097 & 0.8444 & 29.1556 \\
\hline 40 & 0.5622 & 39.4378 & 1.0583 & 38.9417 \\
\hline 50 & 0.6233 & 49.3767 & 1.2626 & 48.7374 \\
\hline 60 & 0.6767 & 59.3233 & 1.4597 & 58.5403 \\
\hline 70 & 0.7243 & 69.2757 & 1.6511 & 68.3489 \\
\hline \multicolumn{5}{|c|}{ 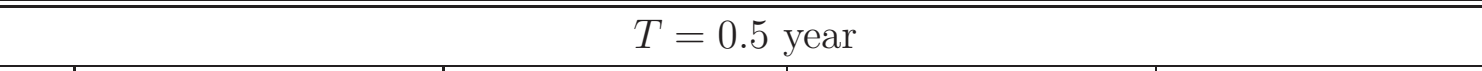 } \\
\hline 30 & 0.3279 & 29.6721 & 0.9274 & 29.0726 \\
\hline 40 & 0.3416 & 39.6584 & 1.1468 & 38.8532 \\
\hline 50 & 0.3502 & 49.6498 & 1.3553 & 48.6447 \\
\hline 60 & 0.3558 & 59.6442 & 1.5557 & 58.4443 \\
\hline 70 & 0.3595 & 69.6405 & 1.7500 & 68.2500 \\
\hline \multicolumn{5}{|c|}{$T=0.75$ year } \\
\hline 30 & 0.2051 & 29.7949 & 0.9560 & 29.0440 \\
\hline 40 & 0.1954 & 39.8046 & 1.1745 & 38.8255 \\
\hline 50 & 0.1863 & 49.8137 & 1.3822 & 48.6178 \\
\hline 60 & 0.1779 & 59.8221 & 1.5820 & 58.4180 \\
\hline 70 & 0.1702 & 69.8298 & 1.7756 & 68.2244 \\
\hline \multicolumn{5}{|c|}{$T=1$ year } \\
\hline 30 & 0.1299 & 29.8701 & 0.9687 & 29.0313 \\
\hline 40 & 0.1138 & 39.8862 & 1.1859 & 38.8141 \\
\hline 50 & 0.1012 & 49.8988 & 1.3925 & 48.6075 \\
\hline 60 & 0.0911 & 59.9089 & 1.5914 & 58.4086 \\
\hline 70 & 0.0827 & 69.9173 & 1.7844 & 68.2156 \\
\hline
\end{tabular}

